\title{
Performance Analysis of Wireless Network Measurement Control System using Matlab/Simulink
}

\author{
Shurui Fan*, Jingbo Li, Jie Li, Hexu Sun \\ Department of Automation, Hebei University of Technology \\ Tianjin, 300130, China \\ * Corresponding author's Email: fansr@hebut.edu.cn
}

\begin{abstract}
Traditional network control system offers many advantages but requires cables to inter-connect devices, which leads to high installation and maintenance costs due to low scalability and high failure rate of connectors. As a result, wireless technologies have gained an enormous success in the consumer goods industry in the last few years. Wireless Networked Measurement Control System (WNMCS) is a distributed control system based on the wireless measurement network, suitable for use at the device level of an automation system. For that purpose, Wireless Local Area Networks (WLAN) and Wireless Personal Area Networks (WPAN) can be employed. In this paper, a simulation model of WNMCS is designed on the basis of Matlab/Simulink, and the suitability of wireless sensor networks for networked control loop is shown. Real time character is the common request of control systems and multitasks is the mainstream of the distributed control system nowadays. Aiming at selecting communication protocol in the wireless networks, the TrueTime toolbox is applied to establishing the simulation model of the Wireless Network Measurement Control System (WNMCS). We compare the performance of WPAN-based WNMCS to that of WLAN-based WNMCS. Experimental results show that WPAN-based WNMCS is a very promising alternative for wireless industrial networking with short superframe, and WLAN-based WNMCS is more suitable for high throughput application.
\end{abstract}

Keywords: Wireless Networked Control System, Real time character, Wireless Personal Area Networks, Truetime

\section{Introduction}

In recent years, the great advances in microelectronics and micro-electro-mechanical system have made available inexpensive smart sensors that are equipped with sensing, data processing and wireless communication capabilities. The proliferation of these products in turn makes it possible to design real-time control loops over wireless networks. Consequently, a new generation of networ- ked control systems called Wireless Networked Measurement Control Systems (WNMCS) is emer- ging.

An overview of technologies available for sensor networking in industrial applications has been presented in paper $[1,2]$. Traditional networking offers many advantages but requires cables to inter-connect devices, which leads to high installation and main- tenance costs due to low scalability and high failure rate of connectors. As a result, wireless technologies have gained an enormous success in the consumer goods industry in the last few years.

The industrial environment is error prone and the reliability of wireless technology is lower than wired one, which makes it difficult to support a real-time transmission and multiple $\mathrm{I} / \mathrm{O}$ nodes in the industrial environment. Therefore, it is necessary to design a WNMCS which can support multiple nodes and guarantee real-time transmission of mixed traffic in an industrial environment. Generally speaking, several media can be exploited, including light and ultrasound, but considerations regarding data size, rates and area coverage make radio frequency (RF) links more attractive. Many standards have been proposed to satisfy requirements of the consumer world, as proved by the IEEE802 subgroups which 
cope with these topics in paper [3, 4].

These standards are studied for the selection of the best suitable for WNMCS. The following part of paper is organized as follows. In section 2, the existing wireless technologies are analyzed. Then, the proposed wireless networks measurement control system is introduced. In Section 4, two scenarios are used to compare IEEE 802.15.4 with IEEE 802.11 b. Section 5 provides simulation results to show the performance of WNMCS and conclusions are presented in Section 6.

\section{The IEEE 802 standards}

Wireless communications are an effective and reliable solution in home and office automation. Generally speaking, several media can be exploited, including light and ultrasound, but considerations regarding data size, rates and area coverage make radio frequency (RF) links more attractive. Many standards have been proposed to satisfy requirements of the consumer world, as proved by the IEEE802 subgroups which cope with these topics.

Some efforts have been made on applying wireless technologies such as IEEE 802.11 and IEEE 802.15 to control systems[5,6]. Some standards are chosen for their diffusion in both industrial and public environments to implement wireless networks.

\subsection{IEEE 802.11}

In 1997, IEEE 802.11 Working Group released the 802.11 protocol, which is the first internationally recognized agreement in the field of wireless LAN. With the development of 802.11, several different standards have emerged such as $802.11 \mathrm{~b} / \mathrm{a} / \mathrm{g}$ standard. There exist the technical and performance differences between them. The former based on the CSMA / CA protocol, providing point to point against the other communication services; the latter controlled by the access point (AP), providing real-time communication services.

IEEE 802.11 is a multiple access protocol in which stations in the network must "compete" for access to the shared communications medium to transmit data. IEEE 802.11 uses, as does Ethernet, a carrier sensing capability to determine if the communications medium is currently being used. If two or more stations in the network transmit at the same time (i.e., a collision occurs), stations retransmit their data after random periods of time as in Ethernet. Since IEEE 802.11 is a WLAN standard, its key intentions are to provide high throughput and a continuous network connection [8].

\subsection{IEEE 802.15}

In the IEEE 802.15 specifications, there are four working groups. The difference between them is the rate of data transmission, power consumption level and quality of service (Quality of Service, QoS).

- TG1: WPAN / Bluetooth Task Group, which is a middle-level transmission rate WPAN, typically used in cell phones and PDA newsletter.

- TG2: Coexistence Task Group, which is to raise compatible mechanisms for the IEEE 802.15.1 and IEEE 802.11 protocol.

- TG3: WPAN High Rate Task Group, which requires a higher transmission rate and service quality, mainly applied to multimedia.

- TG4: WPAN Low Rate Task Group, whose features are same with sensor network such as low energy consumption, lower transmission rates and inexpensive features.

\subsubsection{IEEE 802.15.1}

IEEE $802.15 .1 /$ Bluetooth is a low cost/shortrange (up to $10 \mathrm{~m}$ ) wireless networking method for personal, office and industrial enviro- nments [7]. It is a technical standard for wireless digital communications raised by Ericsson, Nokia, Toshiba, IBM and Intel five companies in May 1998. It can replace the data cable for short-distance communication between the objects. The aim of this Standard was, instead, to unite personal computing devices. Bluetooth has a low consumption but is able to work only in short range. The maximum data rate for Bluetooth is 1 Mbps, using Gaussian binary frequency shift keying (FSK). It supports maximum 7 slave devices controlled by a master. The master always transmits in 'even numbered' time-slots and the slaves transmit in 'odd numbered' timeslots. Each channel is divided into time slots of $625 \mu \mathrm{secs}$. The master switches from slave to slave in a round-robin fashion.

\subsubsection{IEEE 802.15.4}

The goal of IEEE 802.15.4 was to create a very low cost, very low power, two-way wireless communication solution which meets the unique requirements of sensors and control devices $[9,10]$. In contrast to IEEE 802.15.1 and IEEE 802.11, IEEE 802.15.4 has been specifically developed to apply to such a static network which contains many infrequently used devices transmitting only small data packets. In order to encourage widespread deployment, IEEE 802.15.4 has been placed in unlicensed frequency bands. Within these bands, 
direct sequence spread spectrum (DSSS) is used in order to comply with the respective sharing rules of each band as well as to allow for simple analog circuitry to be used. The maximum data rate of the DSSS is $250 \mathrm{Kbps}$ in a single channel within the 2.4 $\mathrm{GHz}$ band. In total, the $2.4 \mathrm{GHz}$ band accommodates 16 such channels.

The devices operating with the IEEE 802.15.4 standard are distinguished in two kinds: full-function devices (FFD) and reduced-function devices (RFD).A FFD can become a network coordinator and work with other FFD in a peer-to-peer fashion.

The RFD, on the other hand, are always associated with one of these FFD and limited to exchanging data with this device alone. All devices have a 64 bit address, but it is possible for RFD to obtain a 16 bit shorthand address from their coordinator FFD.

Bluetooth technology is composed of few nodes and has a small coverage area. Hence, it is difficult to apply in large factory area where multiple nodes are required. IEEE 802.11 nodes are expensive and have reliability limitations. In general, multiple nodes are required in an industrial environment. Therefore, the WNMCS based on IEEE 802.11 is limited by high installation and maintenance costs. IEEE 802.15.4 with non-beaconenabled mode suffers from random delays, frame losses and limited data rate. The beaconenabledmode in IEEE 802.15.4 can offer real-time guarantees by means of the GTS mechanism. Each node of the LR-WPAN is inexpensive. Hence, using the beacon enabled mode makes IEEE 802.15.4 suitable for implementing closed-loop for real-time systems.

\section{Wireless networks measurement control system}

Consider a wireless networks measurement control system as shown in Figure 1, which is described in [11]. There are $\mathrm{N}$ independent control loops. For simplicity, assume each control loop is composed of one sensor, one controller, and one actuator. The sensor and the actuator are attached to the controlled process, which is a single-input singleoutput physical system. All these nodes reside within a collision area in which every pair of nodes can hear from each other, i.e., all nodes share the same wireless channel. The wireless technology used in the network can be IEEE 802.15.4 or IEEE $802.11 \mathrm{~b}$.

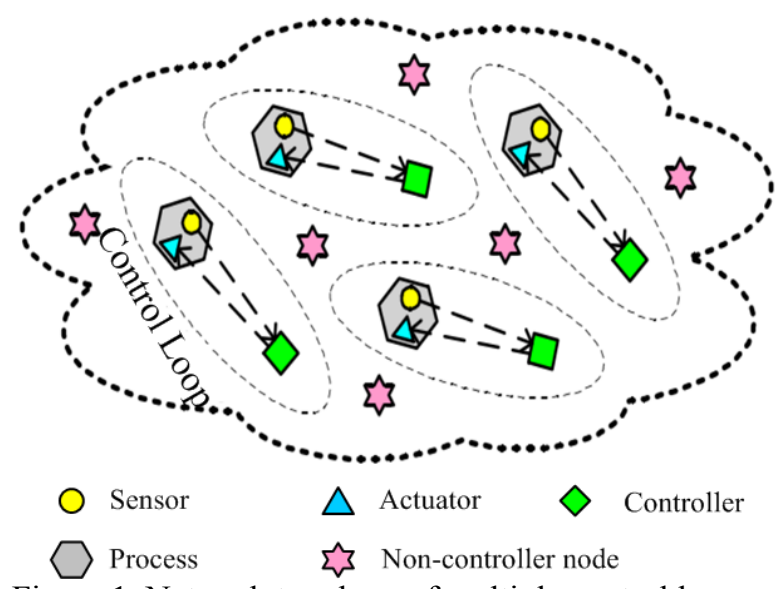

Figure 1. Network topology of multiple control loops within a collision area.

The block diagram of the WNMCS is given in Figure 2. In each control loop, the sensor and the actuator communicate with the controller wirelessly. The sensors are time triggered, while the controllers and the actuators are event triggered. At the beginning of a sampling period, the sensor collects a measurement of the output of physical process, and then transmits it to the controller via wireless channel. During this term, it may need to compete with other coexisting nodes for the use of the network resource. Upon receiving the sampled data, the controller starts to execute the control algorithm immediately. After the control command is produced, the controller will transmit it to the actuator again over the wireless channel. The actuator will perform the corresponding actions on the physical process once it receives the control command.

The controlled process is a DC motor modeled by:

$$
\mathrm{G}(\mathrm{s})=\frac{1000}{\mathrm{~s}^{2}+\mathrm{s}}
$$

As mentioned above, this paper contrasts some wireless standards to determine protocols used in wireless networks measurement control system. Generally speaking, many control algorithms/techniques can be used in this context. In particular, the PD control algorithm is employed in this paper. The PD-controller is implemented according to the following equations:

$$
\begin{aligned}
& P=K \times(r(k h)-y(k h)) \\
& D(k h)=\frac{T_{d}}{N h+T_{d}} D(k h-h)-\frac{K_{d} N}{N h+T_{d}}(y(k h)-y(k h-h)) \\
& u(k h)=P(k h)+D(k)
\end{aligned}
$$

Where:

$\mathrm{K}=$ proportional gain

$\mathrm{Td}=$ derivative time

$\mathrm{N}$ is used to limit the gain for higher frequencies.

$\mathrm{r}=$ the command (reference) signal, acting on 
the proportional part.

$\mathrm{h}$ is the sampling time.

$\mathrm{y}=$ output

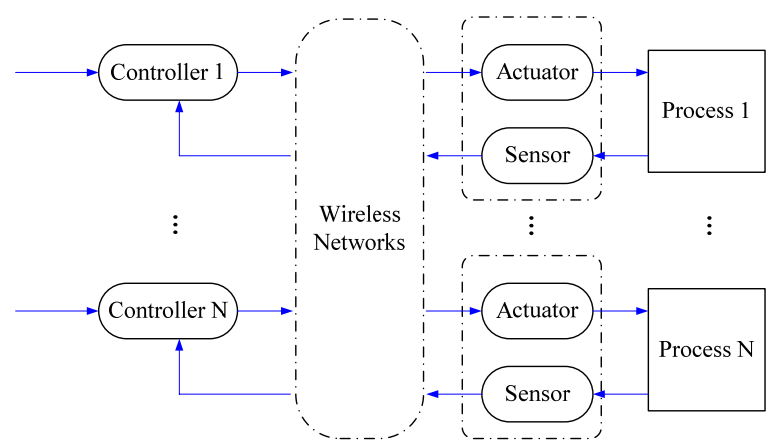

Figure 2. Block diagram of the wireless networks measurement control system

\section{Implementation of Scenario}

In this section, simulations are conducted based on Matlab/Truetime [13 15] to evaluate the performance of IEEE 802.11b and IEEE 802.15.4.

MATLAB is a software program package for technical calculation and data visualization. The result from reliable calculations can be presented with advanced graphics. The program package is flexible, user-friendly, and can be applied to mathematics, physics, engineering, chemistry, and biology. True Time is a Simulink-based simulator written in $\mathrm{C}++$ MEX for real-time control systems. It facilitates the simulations in the following aspects:

- Controller task execution using a multitasking real-time kernel, where the tasks are controlling processes which are modeled as ordinary Simulink blocks

- Simple models of communication networks and their influence on networked control loops

- The TrueTime simulator was developed by Department of Automatic Control, LTH1, Sweden, and commonly used in the following situations:

- Investigation on the effects of timing non-determinism caused by preemption or transmission delays and on control performance.

- Development of compensation schemes which adjust the controller dynamically based on measurements of actual timing variations

- Experiment with new and more flexible approaches to dynamic scheduling, such as feedback scheduling of CPU time and communication bandwidth and quality-of-service (QoS)-based scheduling approach.

- Simulation of event-driven control system such as engine controllers and distributed controllers.

- TrueTime version 2.0 used in this project is delivered as a Simulink library, consisting of the following blocks:

- Kernel block supporting external interruptions, possibility to write tasks as M-files or $\mathrm{C}++$ functions and possibility to call Simulink block diagrams from within the code functions

- Network block with protocol settings available for Ethernet, CAN, TDMA, FDMA, Round Robin, and switched Ethernet networks

- Wireless network block which supports 802.11b/WLAN and 802.15.4/ZigBee Scaling

- Battery block supporting Dynamic Voltage

The three blocks will not be discussed in details. Further information about these blocks can be found in the TrueTime Reference Manual [15]. The porting of the network-related implementation in the kernel block will include only the configuration of network I/O ports (Receive/Send) to maintain the compatibility in future development. In other words, there is no functionality available behind these network $\mathrm{I} / \mathrm{O}$ ports.

The kernel block works as a computer with a simple but flexible real-time kernel equipped with $\mathrm{A} / \mathrm{D}$ and $\mathrm{D} / \mathrm{A}$ converters, a network interface, and external interrupt channels. As common real-time kernels, it maintains data structures for a readyqueue, a time queue, and records for tasks, interrupt handlers, monitors, and timers that have been created for the simulation. The execution of tasks and interrupt handlers is configured by user-defined code functions that can be written in $\mathrm{C}++$ (for speed) or as MATLAB m-files (for ease of use). Control algorithm is modeled graphically using ordinary Simulink block diagram.

Truetime is a Matlab/Simulink-based simulator for real-time control systems. Truetime facilitates co-simulation of controller task execution in realtime kernels, network transmissions, and continuous plant dynamics. Features of the simulator include:

Figure 3 and figure 4 show two scenarios setup in the graphical setup in Simulink.

Two different scenarios were used for the simulations. The first setup is used to experiment with TrueTime and to investigate the possibilities for sending data through the network. The model contains two computer nodes located $10 \mathrm{~m}$ apart, each represented by a TRUETIME kernel. A timedriven sensor/actuator node samples the process periodically and sends the samples over the network to the controller node. The control task in this node calculates the control signal and sends the result back to the sensor/actuator node, where it is actuated. 


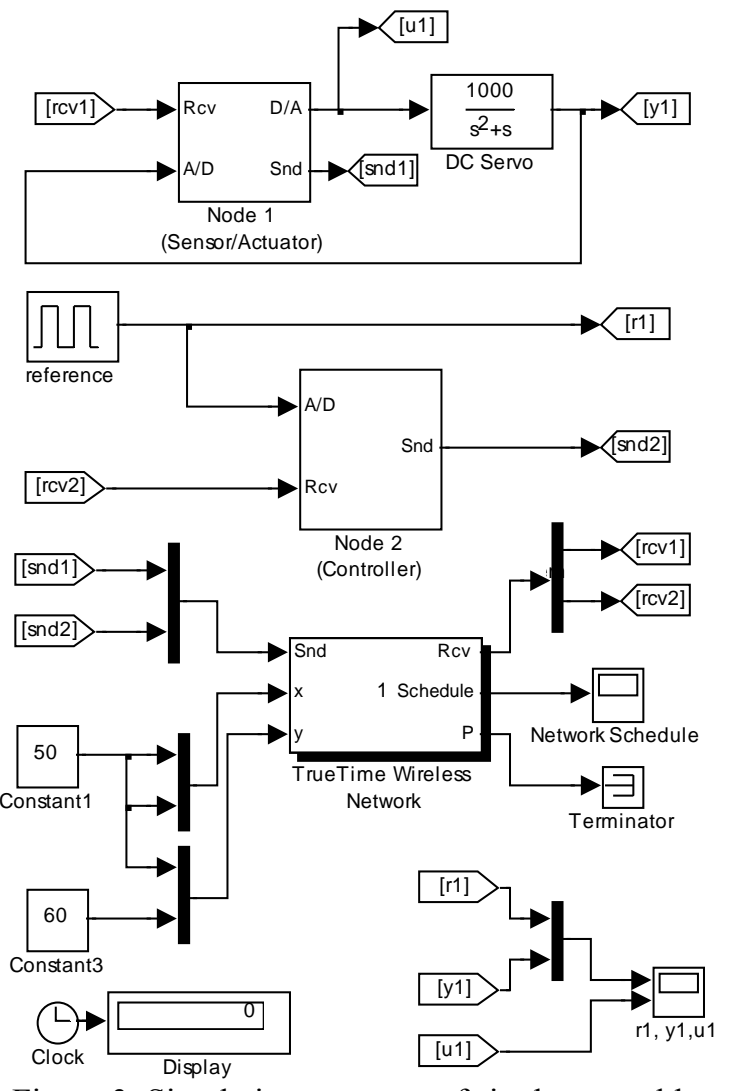

Figure 3. Simulation structure of single control loop based on truetime toolbox
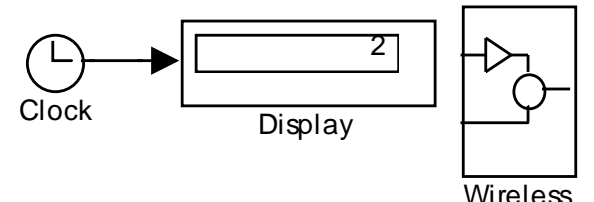
Network

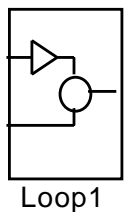

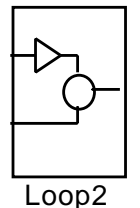
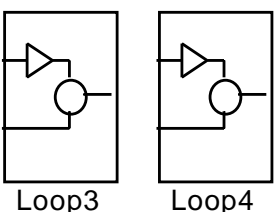

square wave with a period of $2 \mathrm{~s}$. Since the time-triggered sampling scheme is implemented in each control loop separately, the PD parameters can be different from one loop to another. For simplicity, the same parameters are used in all sensors: $\mathrm{h}=$ $10 \mathrm{~ms}, \mathrm{~N}=100000, \mathrm{Td}=0.035$, and $\mathrm{K}=1.5$.

\section{Simulation Case Studies}

\subsection{Study the performance of IEEE 802.11b}

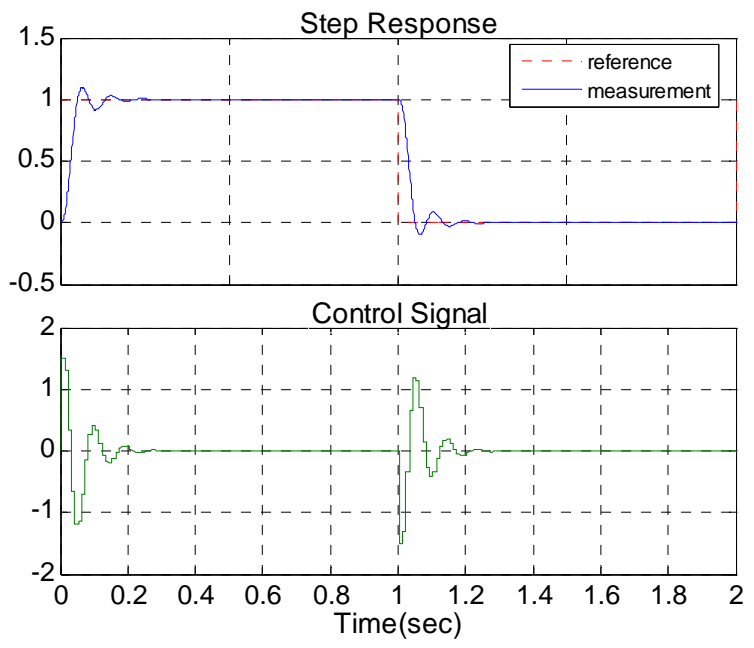

(a) $0.25 \mathrm{Mbps}$ date rate
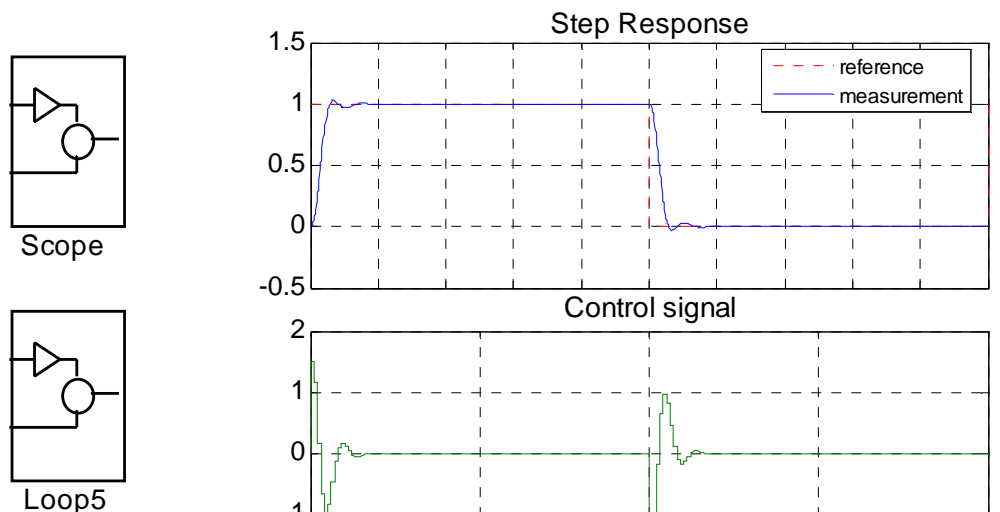

Figure 4. Simulation structure of multi-control loop based on truetime toolbox

The second simulation setup is to a large extent the same as the one shown in figure 4. Loop is the integration of figure 3. Loop1 contain node1 and node2, where node1 is sensor1/actutaor1 and node2 is controller. Loop 2 contain node 3 and node4, where node 3 is sensor/actuator and node4 is controller. Like that, Loop5 contain node9 and node10, where node9 is sensor/actuator and node10 is controller. The position and wireless protocol are set in wireless network block. Both the controlled process and the controller design are kept as common as possible to reflect the wide applicability of the proposed approach. The reference input follows a

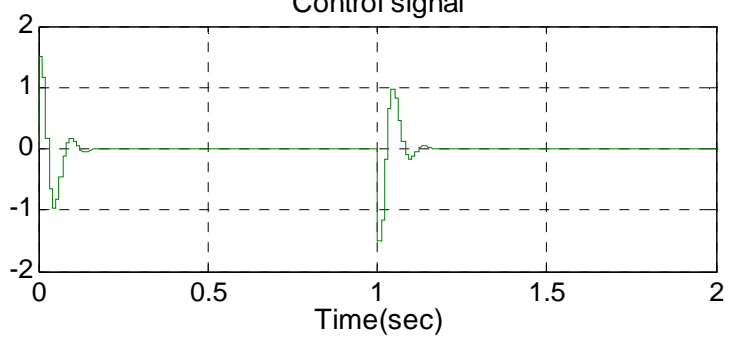

(b) $2.5 \mathrm{Mbps}$ date rate

Figure 5. Single loop Control performance of IEEE $802.11 b$

IEEE $802.11 \mathrm{~b}$ standard is used for testing the affect of different data rate. Figure 5 is the first scenario (figure 3 ) performance curve with the data transfer rates $2.5 \mathrm{Mbps}$ and $0.25 \mathrm{Mbps}$, respectively. The results show that the DC motor output y is able to track the reference input $r$ curve through a wireless network, and system control becomes better when the data transfer rate gets higher.

In Figure 5 (a), when simulated by the 0.25 Mbps date rate in the network control system, the 
system overshoot is about $5 \%$, adjustment time is less than 0.2s. In Figure 5 (b), with 2.5 Mbps setting, there is a remarkable improvement.

\subsection{Study the performance of IEEE 802.15.4}
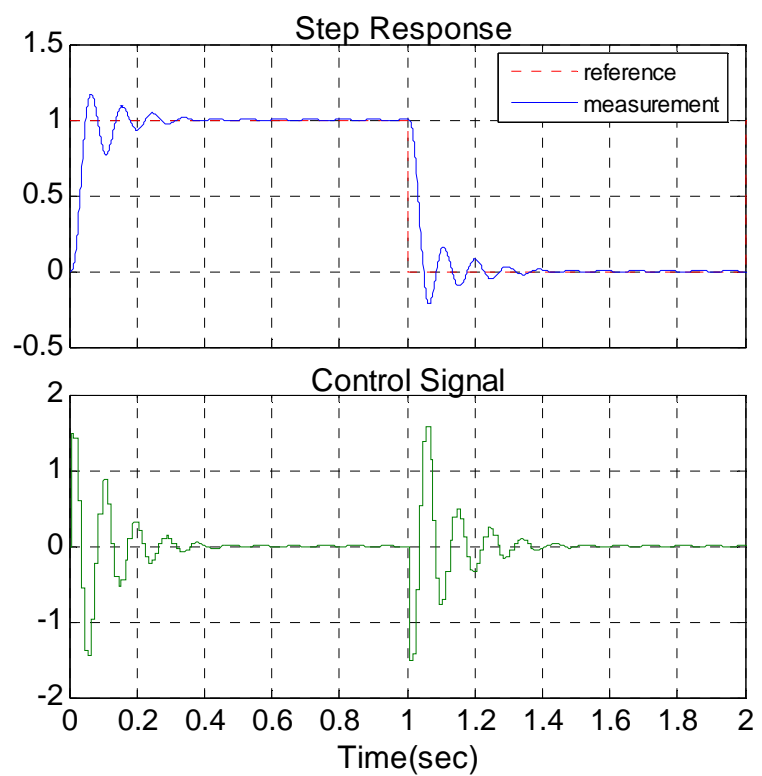

(a) minimum frame size $248 \mathrm{bit}$
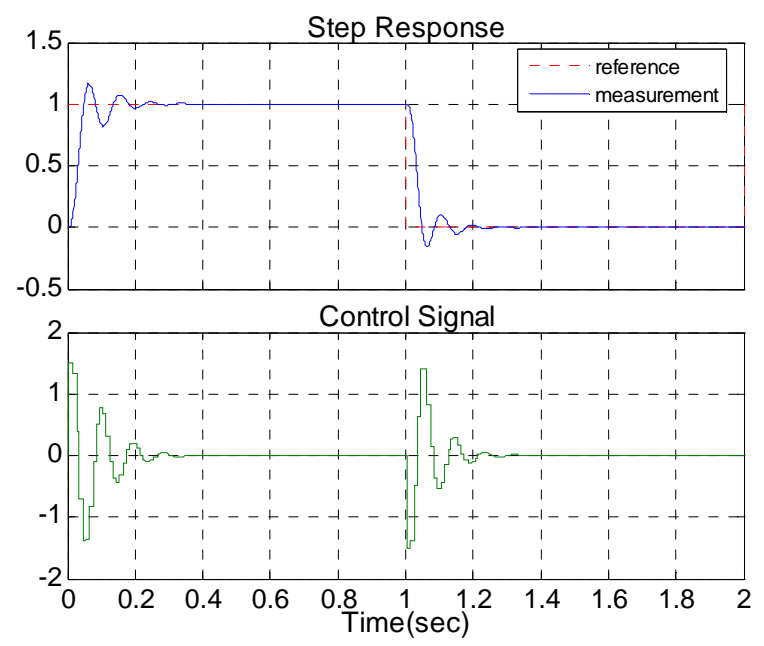

(b) minimum frame size 10bit

Figure6. Single loop Control performance of IEEE 802.15 .4

IEEE 802.15.4 standard is used for testing the performance of single control loop. In the first scenario (Figure 3), we use IEEE 802.11b and IEEE 802.15.4 protocol respectively, and set the same data transfer rate, without power control strategy. Both kinds of control loops achieve good performance, with the same setting of $250 \mathrm{~Kb} / \mathrm{s}$. The control performance of IEEE $802.11 \mathrm{~b}$ is shown in Figure 5(a), for IEEE 802.15.4 is Figure 6(a). In Figure 5(a), the system overshoot is about $5 \%$, adjustment time is less than 0.2s. In Figure 6(a), the system overshoot is about $10 \%$, adjustment time is more than $0.3 \mathrm{~s}$. By reducing the data frame length, the control performance is improved, which is shown in figure $6(b)$.

The results show that using the IEEE $802.11 \mathrm{~b}$ protocol can improve data transfer rate and system performance. In the low data transfer rate, IEEE 802.15.4 protocol is more suitable for short date frame applications with limited power consumption by virtue of its low power characteristics.

\subsection{Multi-loop control system performance}
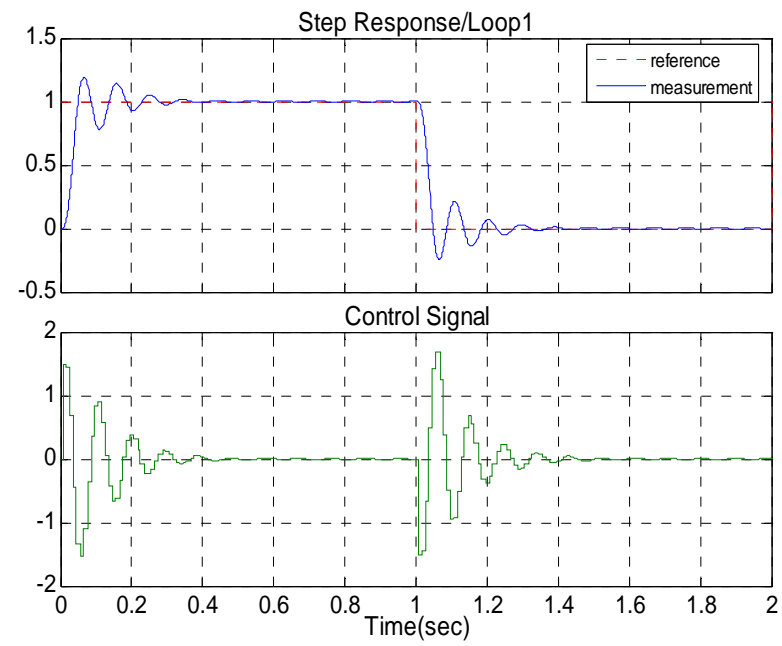

(a) Control performance of Loop1

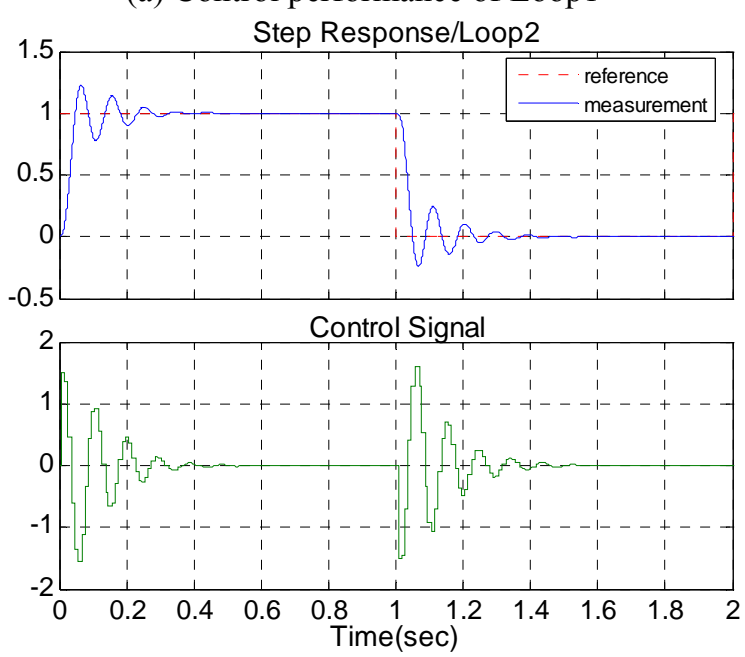

(a) Control performance of Loop2

Figure7. The simulation results corresponding to the scenario described in figure 4

In the second scenario (figure 4), IEEE802.15.4 protocol is selected to build the wireless industry control network. The network schedule and response curve of the control loop1 and loop2 are shown in figure 7 .

The results show that each control loop can work steadily, so that IEEE 802.15.4 communication protocol can be used to form wireless network measurement control system. 
From the simulation curve, multi-loop control system WNMCS has larger overshoot, longer settling time and slower response speed, compared with a single control loop. Specified in 4.1, the higher data transfer rate can improve performance of control system. But the bandwidth of the system is limited for any wireless protocol; network confliction is increased with the enhancement of data rate.

The network scheduling state diagram 8 of the Network scheduling windows shows that Network conflict will occur, however, the network delay has a very good performance. The network schedule has the following representation. Low means that the node is idle and has nothing to send. Medium corresponds to the fact that the node is waiting to transmit, but for some reason has not started yet. For example, the net is busy or it is counting down its back-off timer from previous collisions. And finally, high means that the node is sending.

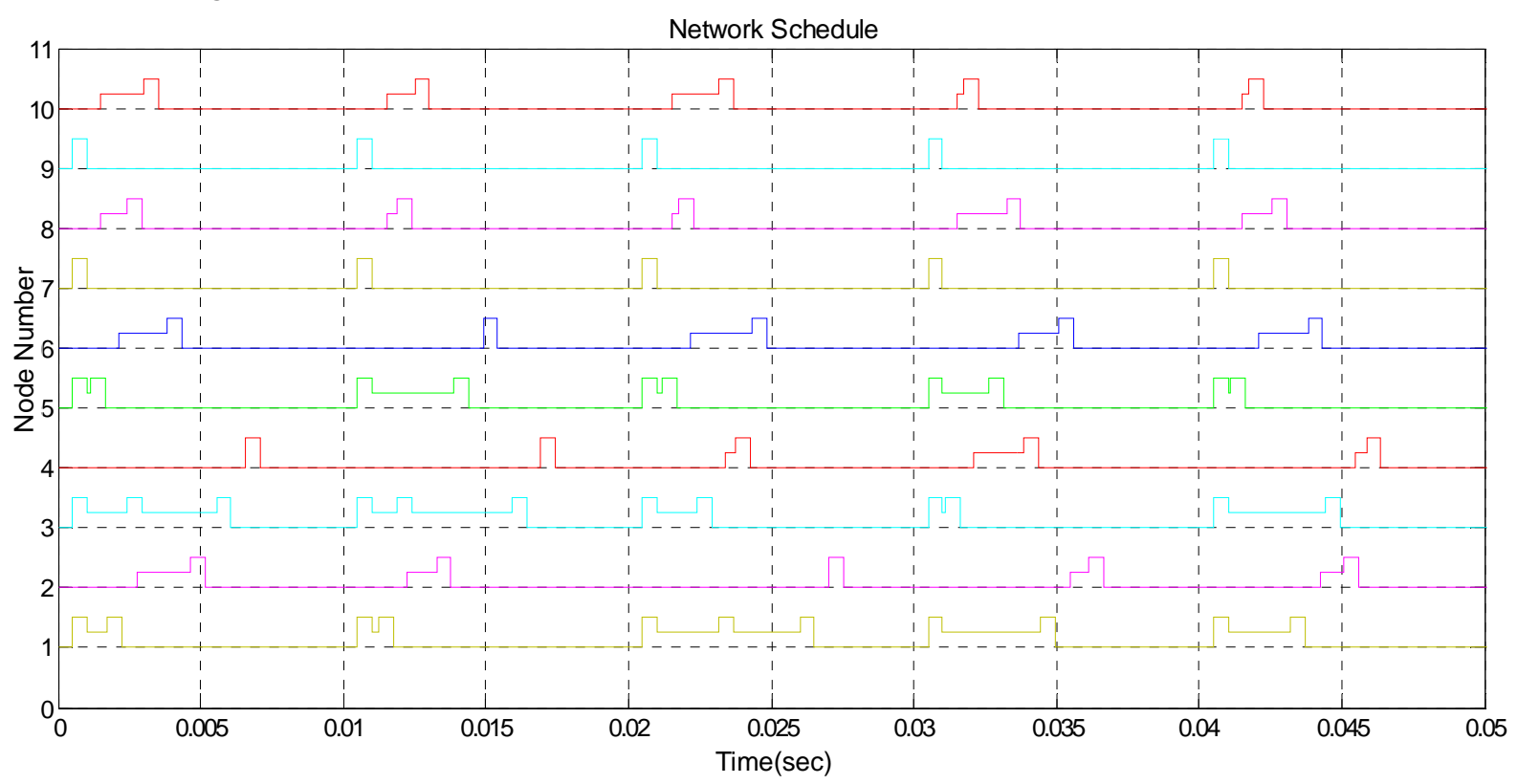

Figure 8. Network schedules corresponding to the scenario described in Figure 4

\section{Conclusion}

With the development of communication and processor technology, wireless network is becoming a strong competition against the wire network. IEEE 802.15.4 is a good choice for short distance, low-rate and real-time control system, with its low-power, low-cost and low-latency. The simulation shows that IEEE 802.15.4 can be used to form wireless network measurement and control system. The industrial environment is composed of a number of sensors, actuators and I/Os. Most transmitted I/O data other than network maintenance data are less than 1 byte [1]. The WNMCS should be able to transmit real-time periodic data, sporadic data for alarm and nonreal-time message data for network maintenance [2]. So the next step is how to use IEEE 802.15.4 to set the more large-scale wireless networks.

\section{References}

[1] A.Flammini, P.Ferrari, D.Marioli. et al, "Wired and wireless sensor networks for industrial applications",
Microelectron, 2008.

[2] Peter Neumann. "Communication in industrial automation-What is going on ?", Control Engineering Practice, Elsevier Ltd, pp.1332-1347, 2007.

[3] D.Hyuk Choi, Dong-Sung Kim, "Wireless Fieldbus for Networked Control Systems using LR-WPAN", International Journal of Control Automation and Systems, Vol.6, No.1, pp. 119 - 125, 2008.

[4] A.Willig, K.Matheus, A.Wolisz, "Wireless Technology in Industrial Networks", Proceedings of the IEEE, Vol.93, No.6, pp.1130-1151, 2005.

[5] Xia,F.;Tian,Y.-C.;Li,Y.;Sun,Y.”Wireless Sensor/Actuator Network Design for Mobile Control Applications", Sensors, Vol.7, pp.2157-2173, 2007.

[6] Pellegrini,F.D.; Miorandi,D.; Vitturi,S.; Zanella,A. "On the Use of Wireless Networks at Low Level of Factory Automation Systems", IEEE Trans. on Industrial Informatics ,Vol.2,No.2, pp.129-143, 2007.

[7] IEEE Computer Society, IEEE 802.15.1 standard: Wireless medium access control(MAC) and physical layer(PHY)specifications for wireless personal area networks(WPANs), IEEE, New York,2005.

[8] IEEE Computer Society, IEEE $802.11 \mathrm{~b}$ standard: Wireless LAN Medium Access Control (MAC)and Physical Layer(PHY)specifications: Higher-Speed 
Physical Layer Extension in the $2.4 \mathrm{GHz}$ Band, IEEE, New York, 1999.

[9] IEEE Computer Society, IEEE 802.15.4 standard: Wireless Medium Access Control and Physical Layer Specifications for Low-Rate Wireless Personal Area Networks, IEEE, New York, 2003.

[10] IEEE Computer Society, IEEE 802.15.4 standard: Wireless Medium Access Control and Physical Layer Specifications for Low-Rate Wireless Personal Area Networks, IEEE, New York, 2006.

[11] Feng Xia, and Wenhong Zhao. "Flexible TimeTriggered Sampling in Smart Sensor-Based Wireless Control Systems", Sensors, Vol.7, pp.2548-2564, 2007.

[12]Chen Peng, Dong Yue, Zhou Gu. "Sampling period scheduling of networked control systems with multiple-control loops", Mathematics and Computers in Simulation, Vol.79,pp.1502-1511, 2009.

[13]Anton Cervin, Martin Ohlin, Dan Henriksson: "Simulation of Networked Control Systems Using TrueTime". www.control.lth.se/truetime.

[14]Daniel Kusnadi. True Time in Scicos, Department of Automatic Control, Lund University, 2007.

[15]Anton Cervin, Dan Henriksson, Martin Ohlin, "TrueTime 1.4 - Reference Manual",Department of Automatic Control, Lund University, September 2006. 